\title{
Adrenalectomía laparoscópica
}

\author{
José De Vinatea ${ }^{1}$, Luis Villanueva ${ }^{1}$, Édgar Gonzales ${ }^{1}$, Augusto Dextre ${ }^{1}$, \\ Freddy Valdivia ${ }^{1}$, Wilber Gallardo ${ }^{1}$, Carmen Asato ${ }^{1}$, Guido Molina ${ }^{1}$
}

Resumen

Palabras clave
Objetivo: Evaluar la efectividad y resultados de la adrenalectomía laparoscópica en la patología adrenal benigna. Materiales y Métodos: Estudio retrospectivo de 15 adrenalectomías realizadas en 13 pacientes por el mismo equipo quirúrgico en el Hospital Nacional Guillermo Almenara Irigoyen EsSalud y en la Clínica San Pablo,. Lima - Perú, entre 1996 y 2005, evaluándose la efectividad y resultados con respecto al tamaño del tumor, técnica quirúrgica y otros aspectos. Resultados: Once adrenalectomías fueron unilaterales y dos bilaterales. Las indicaciones fueron: adenoma no funcionante (2), adenoma funcionante (6), feocromocitoma (3), teratoma (1), leiomiosarcoma (1), mielolipoma (2). No hubo mortalidad ni reoperaciones. Conclusión: La adrenalectomía laparoscópica es factible y segura para tumores menores de $7 \mathrm{~cm}$.

Adrenalectomía; teratoma; leiomiosarcoma; glándulas suprarenales.

\section{Laparoscopic adrenalectomy}

Abstract

Objective: To determine the effectiveness and results of laparoscopic adrenalectomy in the treatment of benign adrenal pathology. Materials and Methods: Clinical characteristics and outcomes of 15 laparoscopic adrenalectomies performed at both Guillermo Almenara Irigoyen National Hospital EsSalud and Clínica San Pablo, Lima - Peru, from 1996 to 2005 were determined, considering tumor size, surgical techniques and others. Results: Eleven unilateral and two bilateral adrenalectomies were indicated for non-functional adenoma (2), functional adenoma (6), pheochromocytoma (3), teratoma (1), myelolipoma (2), and leiomyosarcoma (1). There was no mortality or reoperations. Conclusion: Laparoscopic adrenalectomy for less than $7 \mathrm{~cm}$ benign adrenal tumors is recommended.

Keywords: Adrenalectomy; teratoma; leiomyosarcoma; adrenal glands.

1 Servicio de Cirugía General No 4. Departamento de Cirugía General. Hospital Nacional Guillermo Almenara Irigoyen. EsSalud. Lima. Perú.

\section{INTRODUCCIÓN}

Desde que Gagner realizó la primera adrenalectomía laparoscópica, en $1992\left({ }^{1}\right)$, dicho procedimiento se ha consolidado progresivamente hasta convertirse en el gold standard para los tumores adrenales pequeños $\left({ }^{2}\right)$.

Existen algunos estudios comparativos que muestran las ventajas del abordaje laparoscópico sobre el convencional $\left(^{3-6}\right)$. Las indicaciones actuales para adrenalectomía laparoscópica incluyen las patologías tumorales productoras de hormonas o las asintomáticas.

Los tumores adrenales que se extirpa con mayor frecuencia son: feocromocitomas, adenomas, quistes, hiperplasias, lipomas, mielolipomas, cáncer y una larga miscelánea. Puede realizarse adrenalectomía derecha, izquierda o bilateral.

El tamaño tumoral es un tema en debate. Casi nadie discute que las tumoraciones por debajo de 5 ó $6 \mathrm{~cm}$ deben ser operadas por vía laparoscópica $\left({ }^{7,8}\right)$. La controversia es con las tumoraciones de 
mayor tamaño. Sin embargo, se ha reportado adrenalectomías laparoscópicas en tumores de hasta $15 \mathrm{~cm}\left({ }^{3,9}\right)$.

El objetivo de este trabajo es mostrar nuestra experiencia en adrenalectomía laparoscópica con diversas patologías, tamaños y técnicas.

\section{MATERIALES Y MÉTODOS}

Se trata de un estudio retrospectivo realizado en el Departamento de Cirugía General del Hospital Nacional Guillermo Almenara Irigoyen EsSalud y la Clínica San Pablo, Lima - Perú, desde diciembre de 1996 hasta marzo de 2005, habiéndose practicado 15 adrenalectomías en 13 pacientes.

El cirujano principal y el resto del equipo quirúrgico fue el mismo en el manejo de todos los pacientes.

Se revisó las historias clínicas y se llenó una ficha donde se consignó los datos del pre, trans y postoperatorio.

Los pacientes fueron transferidos del Servicio de Endocrinología, en donde se les realizó los estudios bioquímicos respectivos para evaluar la funcionalidad tumoral: cortisol en plasma, cortisol en orina, prueba de supresión con dexametasona, catecolaminas en plasma, metanefrinas totales, ácido vanilmandélico y electrolitos séricos.

Además, se hizo los estudios por imágenes, tomografía en todos los casos y resonancia magnética en 6 pacientes. A ningún paciente se realizó punción diagnóstica adrenal percutánea en el preoperatorio.

En 5 pacientes, la tumoración adrenal fue encontrada accidentalmente, por presentar dolor abdominal (en el flanco) o lumbalgia, pero sin otra manifestación clínica que sugiriera algún trastorno hormonal.

Un paciente era obeso mórbido (IMC $>40$ $\mathrm{kg} / \mathrm{m}^{2}$ ) (caso 13), 2 pacientes tenían cirugías abdominales previas (casos 6 y 14) y un paciente tuvo dos cirugías de hipófisis y una cirugía de desconexión del eje hipotálamo hipofisiario (sección del tallo) (caso 3).

Dos pacientes tuvieron adrenalectomía bilateral (casos 3 y 10): Cushing y mielolipoma bilateral.

Los pacientes portadores de tumoraciones menores o iguales a $7 \mathrm{~cm}$ fueron sometidos a manejo laparoscópico, exceptuando un caso (mielolipoma derecho de $11 \mathrm{~cm}$ ) y se tuvo que convertir a cirugía laparoscópica mano asistida (HALS: hand assisted laparoscopic surgery) para su extracción (caso 11).

Los tumores mayores de $7 \mathrm{~cm}$ se planificó realizarlos con el sistema HALS. Se excluyó aquellos con diagnóstico de certeza de cáncer adrenal.

La cirugía fue realizada vía laparoscópica transperitoneal en decúbito lateral (Figura 1). El neumoperitoneo fue instalado en forma cerrada la mayoría de veces, con la aguja Veress a nivel de la línea axilar anterior, por debajo del reborde costal. Se usó una óptica de 30 grados y se colocó 3 o 4 trócares, siendo por lo general necesarios 4 trócares en el lado derecho: dos de $10 \mathrm{~mm}$ para óptica y mano derecha del cirujano, dos de $5 \mathrm{~mm}$ para mano izquierda del cirujano y para separar el hígado. En el lado izquierdo, por lo general

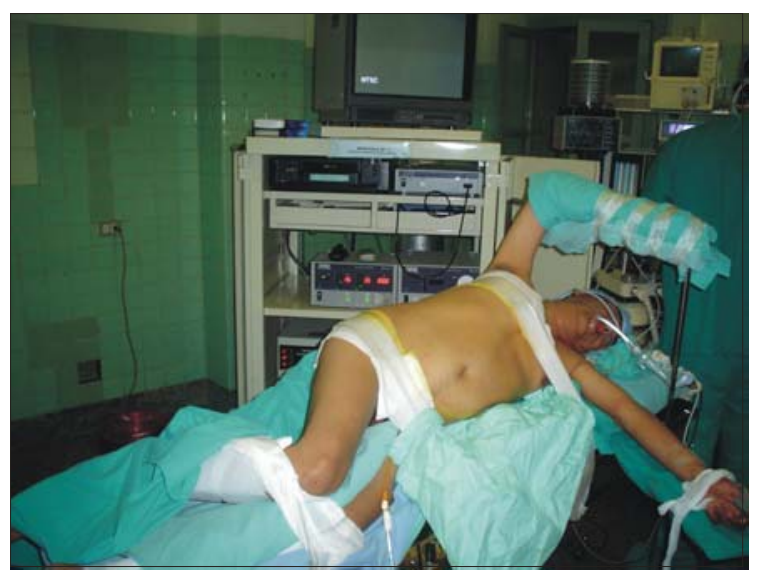

Figura 1. Posición del paciente, adrenalectomía derecha. 


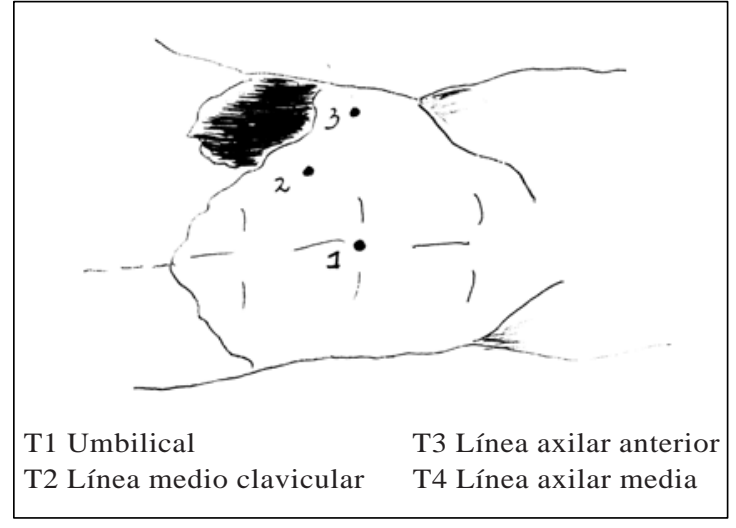

Figura 2. Posición de trocares en adrenalectomía derecha.

fueron suficientes dos de $10 \mathrm{~mm}$ (óptica y mano derecha) y uno de $5 \mathrm{~mm}$ para la mano izquierda del cirujano (Figuras 2 y 3 ).

La vena adrenal fue manejada de diferentes maneras: clips, ligadura intracorpórea, ligasure y ultrasonido. No hubo algún caso de manejo con endo GIA o con coagulación bipolar.

En los casos en que se utilizó el sistema mano asistida, la posición del paciente fue decúbito dorsal con inclinación del lado a extirpar. La incisión de $6,5 \mathrm{~cm}$ fue realizada en forma transversal, infraumbilical. El sistema HALS usado fue el Gelport (Applied Medical) (Figura 4). La mano que ingresó a la cavidad abdominal fue la no dominante del cirujano (mano izquierda). En estos casos, se colocó dos o tres trócares adicionales.

En el lado izquierdo, la disección comienza con la movilización del ángulo esplénico del colon y parte del colon descendente. Se continúa liberando el bazo de sus ligamentos posteriores y superiores, llegando hasta el diafragma. Estando el paciente en decúbito lateral derecho, el colon, el bazo y la cola del páncreas son desplazados hacia la línea media, mostrándose en forma progresiva la glándula adrenal.

Conforme se avanza en la liberación de la glándula, se adquiere mayor movilidad, hasta conseguir visualizar la vena adrenal y proceder

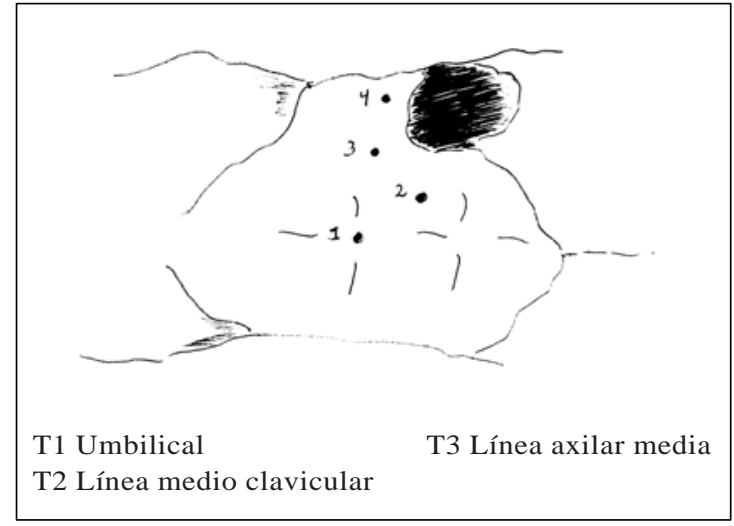

Figura 3. Posición de trocares en adrenalectomía izquierda.

a su sección. El manejo de la vena adrenal izquierda usualmente no reviste mayor dificultad, por su mayor longitud y por su frecuente desembocadura en la vena renal ipsilateral.

En el lado derecho, la disección se inicia abriendo el retroperitoneo a nivel infrahepático. Se diseca hasta llegar a la vena cava inferior medialmente y en el lado externo hasta el ligamento triangular derecho del hígado, el cual también se secciona para que el hígado pueda ser movilizado con facilidad.

Luego de suspender el hígado con la separación del asistente, la glándula es identificada y disecada con maniobras suaves, hasta ubicar la vena adrenal para su respectiva sección. El manejo de la vena adrenal derecha exige mayor cuidado, por su desembocadura directa a la vena cava inferior. Se debe poner especial cuidado en los casos en que la vena adrenal derecha es muy corta, debido al riesgo que representa el pretender reposicionar un clip mal colocado. Al maniobrar una vena corta, se puede lesionar la vena cava, produciéndose una hemorragia severa. En estos casos, está indicada la inmediata conversión para cohibir el sangrado y reparar la lesión, aunque se ha informado la reparación laparoscópica de la vena cava luego de un accidente de este tipo $\left({ }^{2}\right)$.

En todos los casos, la extirpación se realizó en una bolsa, sin morcelar la pieza operatoria. 


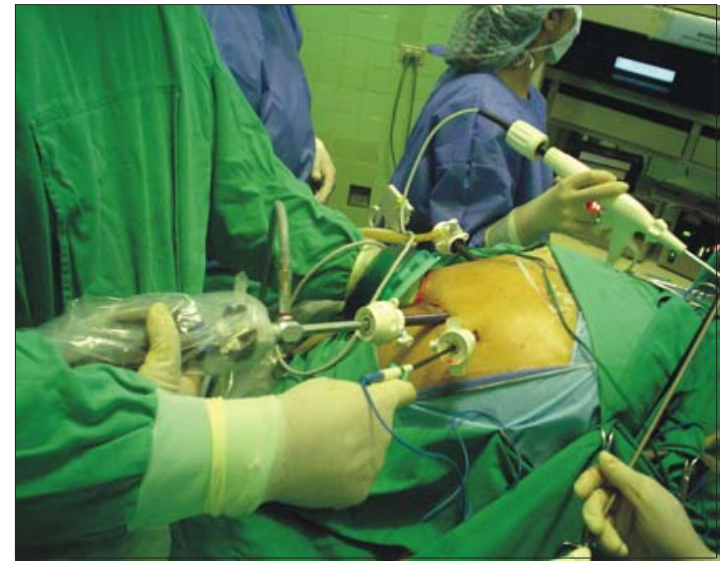

Figura 4. Uso de Gelport (Sistema HALS).

En todos los casos, se utilizó profilaxis antibiótica con una cefalosporina de primera generación. En los pacientes obesos o con cualquier otro factor de riesgo de embolia, se utilizó heparina de bajo peso molecular.

En algunos casos específicos, se utilizó hidrocortisona, la cual fue acompañada de bloqueadores $\mathrm{H} 2$ o inhibidores de la bomba de protones.

No se colocó sonda vesical en forma rutinaria, sino en aquellos casos en los se esperaba una cirugía prolongada (tumoraciones grandes o adrenalectomía bilateral) o con feocromocitoma.

Se usó sonda nasogástrica en el transoperatorio, sobre todo durante la adrenalectomía izquierda. No se dejó drenes en forma rutinaria.

En los casos de feocromocitoma, el manejo fue el siguiente. En el preoperatorio, se administró alfa bloqueadores por vía oral (terazocina) por 3 a 4 semanas y luego beta bloqueadores (propanolol) una semana antes de la cirugía. En el transoperatorio, se contó con un anestesiólogo experimentado en el manejo de dicha patología, quien disponía de las drogas necesarias. Se mantuvo una comunicación permanente entre cirujano y anestesiólogo durante toda la intervención, en alerta a los eventuales cambios hemodinámicos.
Se realizó manejo precoz de la vena adrenal y se minimizó la manipulación tumoral. Se prefirió la suspensión de la glándula sobre la prensión de la misma. También se utilizó la tracción indirecta a través de sus tejidos periféricos, para prevenir la rotura de la cápsula adrenal.

En los casos de aldosteronoma, se usó espironolactona $25 \mathrm{mg}$ diarios, inicialmente, subiéndose la dosis progresivamente hasta llegar a $100 \mathrm{mg}$ durante 8 semanas antes de la cirugía.

En el postoperatorio, se inició la vía oral al momento de presentarse ruidos hidroaéreos o una adecuada eliminación de flatos. Usualmente, ocurrió a la mañana siguiente de la cirugía. Luego de comprobar una adecuada ingesta, se retiró los sueros y se pasó a administrar toda la medicación por vía oral. Se inició la deambulación en forma precoz y se administró analgesia no narcótica por vía oral.

\section{RESULTADOS}

Desde diciembre de 1996 hasta marzo de 2005, se operó 13 pacientes, practicándose 15 adrenalectomías laparoscópicas. Fueron 9 mujeres y 4 hombres, con un promedio de edad de 44 años (Tabla 1),

Tabla 1. Distribución de pacientes de acuerdo a diagnóstico, edad y sexo.

\begin{tabular}{lcc}
\hline \multicolumn{1}{c}{ Diagnóstico } & Sexo & Edad (años) \\
\hline 1. Feocromocitoma & $\mathrm{F}$ & 63 \\
2. Leiomiosarcoma & $\mathrm{F}$ & 56 \\
3. Adenoma funcionante & $\mathrm{F}$ & 23 \\
4. Adenoma funcionante & $\mathrm{F}$ & 23 \\
5. Teratoma maduro & $\mathrm{M}$ & 15 \\
6. Adenoma no funcionante & $\mathrm{M}$ & 69 \\
7. Adenoma funcionante & $\mathrm{F}$ & 29 \\
8. Adenoma funcionante & $\mathrm{F}$ & 43 \\
9. Feocromocitoma & $\mathrm{F}$ & 20 \\
10. Mielolipoma & $\mathrm{M}$ & 56 \\
11. Mielolipoma & $\mathrm{M}$ & 56 \\
12. Adenoma funcionante & $\mathrm{F}$ & 91 \\
13. Adenoma funcionante & $\mathrm{F}$ & 50 \\
14. Feocromocitoma & $\mathrm{M}$ & 27 \\
15. Adenoma no funcionante & $\mathrm{F}$ & 35 \\
\hline
\end{tabular}


Siete casos fueron del lado derecho, Cuatro del izquierdo y dos bilaterales. En 3 casos se instaló el sistema mano asistida (HALS). Se convirtió 3 casos: uno de laparoscopia a HALS (caso 10), uno de laparoscopia a cirugía abierta (caso 14), uno de HALS a cirugía abierta (caso 5). No hubo conversión en los pacientes con tumoraciones menores de $7 \mathrm{~cm}$.

El tamaño tumoral ha sido comparado según imágenes (TAC o RMN), medición en fresco por el cirujano y el tamaño indicado por el informe de anatomía patológica (Tabla 2 ).

Los datos sobre antecedentes y presentación clínica, se muestra en la Tabla 3.

Los datos en relación al tiempo operatorio, necesidad de transfusiones, inicio de vía oral y estancia hospitalaria, se observa en la Tabla 4. La estancia hospitalaria promedio se incrementó por un paciente complicado.

Una paciente (caso 13) presentó un cuadro de neumonía, derrame pleural y celulitis en el miembro inferior izquierdo, que motivó una hospitalización prolongada. Fue una paciente con Cushing, obesidad mórbida y ya había

Tabla 2. Tamaño tumoral comparativo: imágenes, hallazgo quirúrgico y pieza fijada en formol.

\begin{tabular}{lrrr}
\hline \multicolumn{1}{c}{ Diagnóstico } & Tam. I & Tam. AP & Tam. Qx \\
\hline 1. Feocromocitoma & $6 \mathrm{~cm}$ & $5 \mathrm{~cm}$ & $5 \mathrm{~cm}$ \\
2. Leiomiosarcoma & $8 \mathrm{~cm}$ & $6 \mathrm{~cm}$ & $10 \mathrm{~cm}$ \\
3. Adenoma funcionante & $3 \mathrm{~cm}$ & $4 \mathrm{~cm}$ & $4 \mathrm{~cm}$ \\
4. Adenoma funcionante & $4 \mathrm{~cm}$ & $4 \mathrm{~cm}$ & $5 \mathrm{~cm}$ \\
5. Teratoma maduro & $12 \mathrm{~cm}$ & $11 \mathrm{~cm}$ & $12 \mathrm{~cm}$ \\
6. Adenoma no funcionante & $4 \mathrm{~cm}$ & $4 \mathrm{~cm}$ & $3 \mathrm{~cm}$ \\
7. Adenoma funcionante & $3 \mathrm{~cm}$ & $3 \mathrm{~cm}$ & $3 \mathrm{~cm}$ \\
8. Adenoma funcionante & $6 \mathrm{~cm}$ & $5,5 \mathrm{~cm}$ & $5 \mathrm{~cm}$ \\
9. Feocromocitoma & $6 \mathrm{~cm}$ & $6 \mathrm{~cm}$ & $6 \mathrm{~cm}$ \\
10. Mielolipoma & $11 \mathrm{~cm}$ & $10 \mathrm{~cm}$ & $10 \mathrm{~cm}$ \\
11. Mielolipoma & $3 \mathrm{~cm}$ & $4 \mathrm{~cm}$ & $4 \mathrm{~cm}$ \\
12. Adenoma funcionante & $5 \mathrm{~cm}$ & $6 \mathrm{~cm}$ & $5 \mathrm{~cm}$ \\
13. Adenoma funcionante & $6 \mathrm{~cm}$ & $6 \mathrm{~cm}$ & $6 \mathrm{~cm}$ \\
14. Feocromocitoma & $12 \mathrm{~cm}$ & $12 \mathrm{~cm}$ & $14 \mathrm{~cm}$ \\
15. Adenoma no funcionante & $7 \mathrm{~cm}$ & $8 \mathrm{~cm}$ & $7,5 \mathrm{~cm}$ \\
\hline
\end{tabular}

Tam: tamaño

I: imagen

AP: anatomía patológica presentado el derrame en el preoperatorio. Fue evaluada para descartar neoplasia (primaria o metastásica) o alguna enfermedad infecciosa (tuberculosis, VIH, otras). Al descartarse dichas patologías y no mejorar su cuadro respiratorio por la presencia de la patología de fondo, se procedió a la cirugía con las complicaciones ya mencionadas. Salió de alta y en la actualidad se encuentra en buenas condiciones

La respuesta a la cirugía fue buena en todos los casos de tumores funcionantes, con abolición completa de los síntomas. Todos los casos de feocromocitoma respondieron favorablemente. Ninguno utiliza antihipertensivos en la actualidad.

La morbilidad fue de 3 casos (20\%), no hubo reoperaciones y la mortalidad fue cero.

El paciente portador de leiomiosarcoma ha sido considerado como un tumor primario, basado en las características histológicas, a la ausencia de otro foco demostrable en su hospitalización y a lo largo de su evolución, con un seguimiento de 40 meses (Figuras 5, 6, 7 y 8; TAC, RMN, pieza operatoria y microfotografía).

El paciente portador de teratoma tiene un seguimiento de 36 meses, encontrándose asintomático y en buenas condiciones (Figuras 9, 10 y 11 de $\mathrm{RMN}$, pieza operatoria y microfotografía).

\section{DISCUSIÓN}

Las ventajas del abordaje laparoscópico de la patología adrenal son las mismas que para cualquier otro procedimiento laparoscópico: menos dolor, recuperación más rápida, menor agresión, entre otros. Sobre estas ventajas se añaden las específicas de cada patología adrenal: obesidad, inmunosupresión, manipulación.

Si la colecistectomía laparoscópica desplazó rápidamente a la convencional por sus resultados postoperatorios evidentemente superiores, se puede comprender fácilmente el mismo 
Tabla 3. Distribución de pacientes según diagnóstico, antecedentes y presentación clínica.

\begin{tabular}{lll}
\hline \multicolumn{1}{c}{ Diagnóstico } & \multicolumn{1}{c}{ Antecedentes } & \multicolumn{1}{c}{ Presentación Clínica } \\
\hline 1. Feocromocitoma & HTA, dislipidemia & Cefalea, sudoración \\
2. Leiomiosarcoma & DM, HTA, asma & Dolor abdominal \\
3. Adenoma funcionante & Qx. hipofisis, Qx eje H-H & Cushing \\
4. Adenoma funcionante & Qx. hipofisis, Qx eje H-H & Cushing \\
5. Teratoma maduro & Sin importancia & Dolor lumbar \\
6. Adenoma no funcionante & Linfoma, Ca colon, Ca prostata & Asintomático \\
7. Adenoma funcionante & HTA, hiperaldosteronismo primario & Cefalea, disminución fuerza muscular \\
8. Adenoma funcionante & HTA & Cushing \\
9. Feocromocitoma & HTA & Cefalea, palpitaciones \\
10. Mielolipoma & Sin importancia & Asintomático \\
11. Mielolipoma & Sin importancia & Asintomático \\
12. Adenoma funcionante & HTA & Cushing \\
13. Adenoma funcionante & HTA & Cushing + obesidad mórbida \\
14. Feocromocitoma & HTA, nódulo tiroides & Cefalea, palpitaciones \\
15. Adenoma no funcionante & Sin importancia & Asintomático \\
\hline HTA: hipertensión & DM: diabetes mellitus & Qx: cirtugía de \\
H-H: hipotálamo-hipófisis & Ca: cáncer &
\end{tabular}

fenómeno en la cirugía de adrenales. Si además le añadimos que la incisión en la cirugía convencional de adrenales es más grande que la convencional para la vesícula y además que la patología adrenal se acompaña con frecuencia de obesidad, las diferencias son más notorias. Siendo una patología poco frecuente, debe ser reservada para centros especializados, sobre todo los tumores grandes, gran reto para el cirujano.

Consideramos algunos aspectos técnicos importantes. La exploración laparoscópica inicial del abdomen se ve notablemente afectada en la posición lateral, más aún si el paciente es obeso.

La dificultad operatoria observada en algunos casos no está exclusivamente en el tamaño tumoral sino en su relación con estructuras vecinas y su capacidad de ser movilizadas con los instrumentos convencionales de laparoscopia.

El tipo constitucional del paciente también es importante. Los tumores en pacientes delgados son más sencillos de extirpar que en un obeso mórbido.

Por ejemplo, un tumor de adrenal derecha de $5 \mathrm{~cm}$ pero que crece hacia la región posterior de la cava puede ser más difícil de manejar que uno de $8 \mathrm{~cm}$ del mismo lado, pero que su crecimiento es hacia la parte externa o inferior.

En el lado derecho, el factor de conversión está en función de la relación de la glándula con la cava y con el hígado. La glándula derecha es más retrohepática que suprarrenal.

El tipo de tumor también tiene importancia. Un feocromocitoma tiene más dificultad que un mielolipoma o un lipoma. Probablemente, haya menos dificultad en un mieolipoma grande que en un feocromocitoma más pequeño.

Tenemos el caso del mielolipoma del lado derecho, de $11 \mathrm{~cm}$ (Figura 12), en el que se realizó la laparoscopia sin dificultad en toda la parte inferior, medial y posterior del tumor, incluyendo la sección de la vena adrenal y sus arterias. Sin embargo, fue imposible 'enuclear' el tumor del hígado con los instrumentos romos convencionales. Tuvimos que instalar el sistema HALS y en escasos segundos se completó la extirpación del tumor.

En términos generales, nuestra opinión es que se puede extirpar tumores adrenales más grandes en el lado izquierdo que en el derecho. 
Por todas estas razones, descalificar a un paciente para abordaje laparoscópico basado exclusivamente en el tamaño tumoral, creemos que es insuficiente. Existen algunos factores que se puede anticipar en el preoperatorio, como obesidad o ubicación y tipo de tumor. Pero, hay otros factores que solo podrán ser verificados durante el acto quirúrgico, como crecimiento tumoral, longitud de vena adrenal, entre otros.

La intención de la comparación de tamaños según imágenes, pieza en fresco y pieza fijada con formol es para tratar de estandarizar las mediciones. El tamaño tumoral es fundamental para definir, entre otras cosas, si es de manejo laparoscópico, mano asistida (HALS) o por cirugía convencional. El tamaño además ayudará a decidir la conducta a seguir en los 'incidentalomas', y podrá orientar el diagnóstico de cáncer adrenal; ya que el cáncer adrenal está vinculado a los tumores mayores de $10 \mathrm{~cm}$.
En el presente trabajo, solo en tres casos coincidieron las tres mediciones. En el resto se encontró diferencias hasta de $4 \mathrm{~cm}$.

Las diferencias de tamaño podrían ser explicadas de diferente manera: intervalo prolongado entre imagen y cirugía, hemorragia intratumoral, error al medir por cortes tomográficos una estructura no esférica, error al medirlo en sala de operaciones o error al medirlo en anatomía patológica.

El tamaño operatorio (en fresco) es un tamaño tumoral avascular, que en teoría debería de ser menor que el de las imágenes. El tamaño, según medición del patólogo, es un tamaño fijado en formol, lo que debería significar que es menor a los dos anteriores. Sin embargo, los resultados encontrados son muy variables. No hallamos una explicación convincente a este fenómeno.

En la presente serie, cabe resaltar la presencia de 2 patologías no muy frecuentes: teratoma

Tabla 4. Distribución de pacientes por diagnóstico, tiempo operatorio, necesidad de transfusiones, inicio de vía oral, estancia hospitalaria y complicaciones.

\begin{tabular}{|c|c|c|c|c|c|}
\hline Diagnóstico & $\begin{array}{c}\text { Tiempo } \\
\text { Qx. }\end{array}$ & Transfusiones & $\begin{array}{c}\text { Inicio } \\
\text { VO } \\
\text { (días) }\end{array}$ & $\begin{array}{c}\text { Estancia } \\
\text { hospitalaria }\end{array}$ & Complicaciones \\
\hline 1. Feocromocitoma & $3 \mathrm{~h}$ & No & PO2 & 6 & Ninguna \\
\hline 2. Leiomiosarcoma & 2 h 55 min 25 min & No & PO1 & 5 & Ninguna \\
\hline 3. Adenoma funcionante & $1 \mathrm{~h} 30 \mathrm{~min}$ & No & PO2 & 4 & Ninguna \\
\hline 4. Adenoma funcionante & $1 \mathrm{~h} 20 \mathrm{~min}$ & No & PO2 & 4 & Ninguna \\
\hline 5. Teratoma maduro & $5 \mathrm{~h}$ & No & PO2 & 13 & $\begin{array}{l}\text { Colección lecho, } \\
\text { reabsorción espontánea }\end{array}$ \\
\hline 6. Adenoma no funcionante & $2 \mathrm{~h} 50 \mathrm{~min} 20 \mathrm{~min}$ & No & PO1 & 8 & Ninguna \\
\hline 7. Adenoma funcionante & $1 \mathrm{~h} 35 \mathrm{~min}$ & No & $\mathrm{PO} 2$ & 5 & Ninguna \\
\hline 8. Adenoma funcionante & 2 h 25 min & No & PO2 & 3 & Ninguna \\
\hline 9. Feocromocitoma & $2 \mathrm{~h} 40 \mathrm{~min}$ & No & PO2 & 6 & Ninguna \\
\hline 10. Mielolipoma & $2 \mathrm{~h} 30 \mathrm{~min}$ & No & PO2 & 5 & Ninguna \\
\hline 11. Mielolipoma & $2 \mathrm{~h}$ & No & PO1 & 3 & Ninguna \\
\hline 12. Adenoma funcionante & $1 \mathrm{~h} 15 \min$ & No & PO2 & 4 & Ninguna \\
\hline 13. Adenoma funcionante & $2 \mathrm{~h} 50 \mathrm{~min} 15 \mathrm{~min}$ & No & PO1 & $28 *$ & $\begin{array}{l}\text { Atelectasia, neumonía, } \\
\text { derrame pleural, celulitis }\end{array}$ \\
\hline 14. Feocromocitoma & $4 \mathrm{~h} 30 \mathrm{~min}$ & No & PO5 & 8 & Íleo prolongado \\
\hline 15. Adenoma no funcionante & 2 h 20 min & No & PO1 & 4 & Ninguna \\
\hline
\end{tabular}

Qx: quirúrgico VO: vía oral PO: postoperatorio

* Paciente fue intervenida teniendo ya derrame pleural y se realizó toracocentesis antes de la operación. 


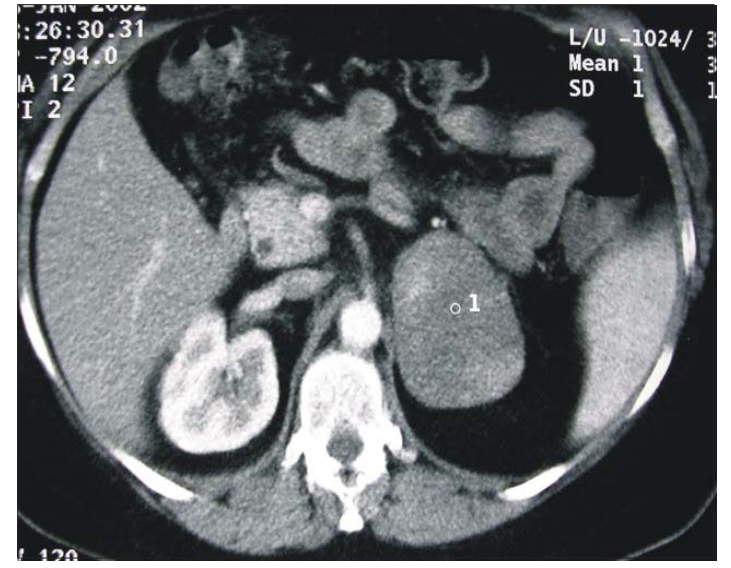

Figura 5. TAC: Leiomiosarcoma adrenal izquierdo.

adrenal y leiomiosarcoma adrenal primario. Ambos fueron un hallazgo. El primero ingresó con el diagnóstico de mielolipoma o lipoma. Esto, debido a la densidad grasa detectada por las imágenes, que correspondió a tejido sebáceo.

El leiomiosarcoma es mucho más raro e ingresó con el diagnóstico de tumoración no funcionante (adenoma). Existen pocos casos reportados en la literatura $\left({ }^{10}\right)$.

Nuestra actitud frente al incidentaloma ha sido la siguiente: solo se procede a su extirpación si

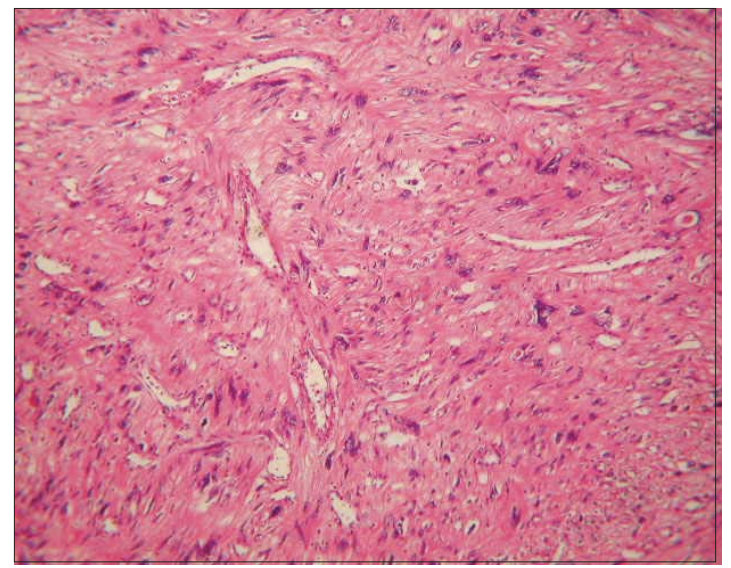

Figura 7. Microfotografía de tumor adrenal mostrando leiomiosarcoma, tinción hematoxilina-eosina.

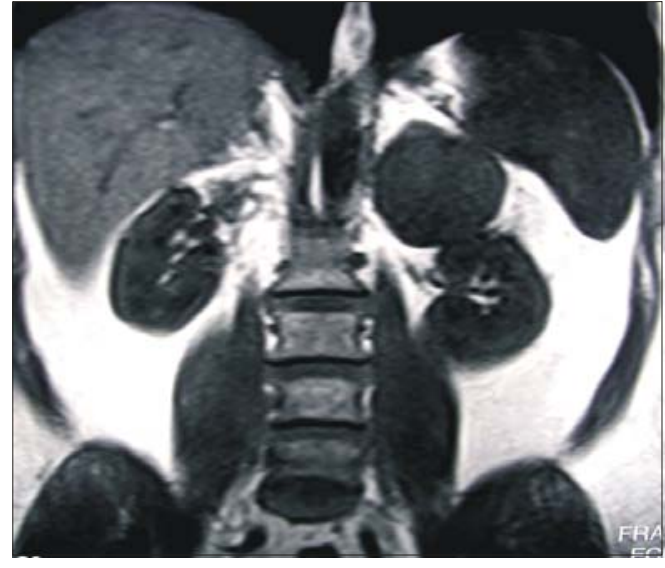

Figura 6. RMN: Leiomiosarcoma adrenal izquierdo.

es que tiene una tamaño mayor de $4 \mathrm{~cm}$, o de cualquier tamaño pero con antecedentes personales de neoplasia. Individualizamos los casos de bilateralidad. Algunos tumores menores podrían ser extirpados si es que no podrán ser controlados regularmente en el futuro.

Tenemos un paciente (caso 6) que tenía antecedentes de triple cáncer: linfoma, cáncer de colon y cáncer de próstata. Se encontró un incidentaloma de $4 \mathrm{~cm}$. Se procedió a su extirpación, siendo un adenoma de $3 \mathrm{~cm}$. Consideramos que fue una decisión adecuada.

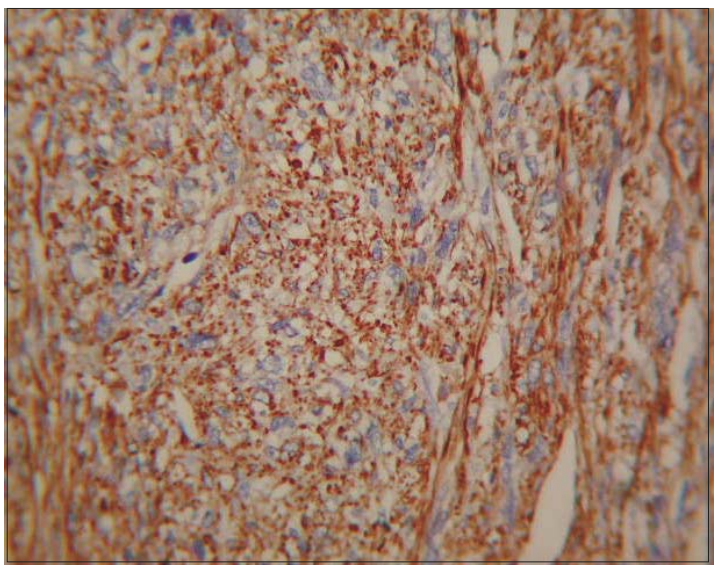

Figura 8. Leiomiosarcoma adrenal (tinción actina). Hallazgos inmunohistoquímicos mostrando reactividad positiva a actina de músculo liso. 
El paciente con mielolipoma bilateral fue operado por el gran tamaño del tumor del lado derecho $(11 \mathrm{~cm})$ y por la duda diagnóstica en el lado izquierdo (presencia de calcificaciones), a pesar de tener solo $3 \mathrm{~cm}$.

Hubo un paciente (caso 3) que había sido intervenido previamente de la hipófisis por un Cushing de origen central. Luego de tres cirugías fallidas, una de las cuales fue una sección del tallo, recién se consideró la alternativa de una adrenalectomía bilateral, la cual fue realizada con éxito con la consiguiente desaparición del Cushing.

Sería interesante para los casos dudosos de Cushing, en los que no se sabe con certeza si es de origen central o periférico, evaluar qué es menos agresivo: iniciar con la cirugía de hipófisis o con la adrenalectomía bilateral laparoscópica.

Si se excluye a la paciente que presentó la estancia prolongada y algunas complicaciones, algunas de las cuales ya las tenía en el preoperatorio, las complicaciones han sido mínimas. La ausencia de mortalidad y de reoperaciones la ratifican como una técnica segura. Esto es principalmente destacable si se recuerda que esta patología va acompañada de obesidad, inmunosupresión y alteraciones hemodinámicas severas.

Tres casos fueron convertidos. Uno a HALS (mielolipoma de $10 \mathrm{~cm}$ ), el segundo de HALS a cirugía convencional (teratoma maduro de 12 $\mathrm{cm}$ por compromiso de vena renal) y otro a cirugía convencional (feocromocitoma de 14 $\mathrm{cm}$ ), por razones técnicas. No hubo conversiones en los tumores menores de $7 \mathrm{~cm}$.

El manejo del feocromocitoma exige el convencimiento de que la clave del éxito radica en una adecuada preparación preoperatoria (bloqueo farmacológico) y contar con el material adecuado para el transoperatorio, incluyendo un anestesiólogo entrenado.

Durante la cirugía, hay que evitar la rotura de la cápsula adrenal, para que no exista el implante celular (feocromocitomatosis) y evitar la recurrencia de la enfermedad $\left({ }^{11}\right)$. Estos cuidados también son aplicables al Cushing, para impedir el implante de células corticales $\left({ }^{2}\right)$.

En algunos casos, el diagnóstico de malignidad primaria está en función de una adecuada interpretación de la relación del parénquima con su entorno más que con la celularidad propiamente, motivo por el cual es muy recomendable la indemnidad de la pieza operatoria. La extracción debe hacerse siempre en bolsa.

El tema del manejo laparoscópico del cáncer adrenal primario o metastásico sigue siendo controversial. Existen series de adrenalectomía laparoscópica para el cáncer adrenal $\left({ }^{12}\right)$, sin recurrencia local ni en las puertas de ingreso, concluyéndose que se puede mantener los principios oncológicos de la cirugía abierta. Las recurrencias loco-regionales tardías son semejantes a la cirugía abierta $\left({ }^{13}\right)$. El uso del sistema HALS extendería aún más las indicaciones de la adrenalectomía laparoscópica para tumores malignos grandes $\left({ }^{14}\right)$. En nuestra serie, no tenemos ningún caso de cáncer diagnosticado preoperatoriamente $\left({ }^{15,16}\right)$. El único caso de neoplasia fue el leiomiosarcoma como hallazgo histológico en el postoperatorio. Por su rareza (imposibilidad de realizar comparaciones adecuadas) y por su diseminación básicamente hematógena, se concluyó que no era necesario ampliar la resección.

Se puede concluir que la adrenalectomía laparoscópica es la mejor indicación para tumores de un tamaño igual o menor a $7 \mathrm{~cm}$. La conversión está en función del tamaño, tipo de tumor, relaciones y ubicación. La laparoscopia y HALS son técnicas seguras en extirpación de tumores adrenales. El empleo del dispositivo mano asistida (HALS) permite la extirpación de tumores de mayor tamaño y complejidad. Siendo una patología poco frecuente, debería ser centralizada en lugares donde se cuente con el equipo quirúrgico entrenado y la infraestructura adecuada. 


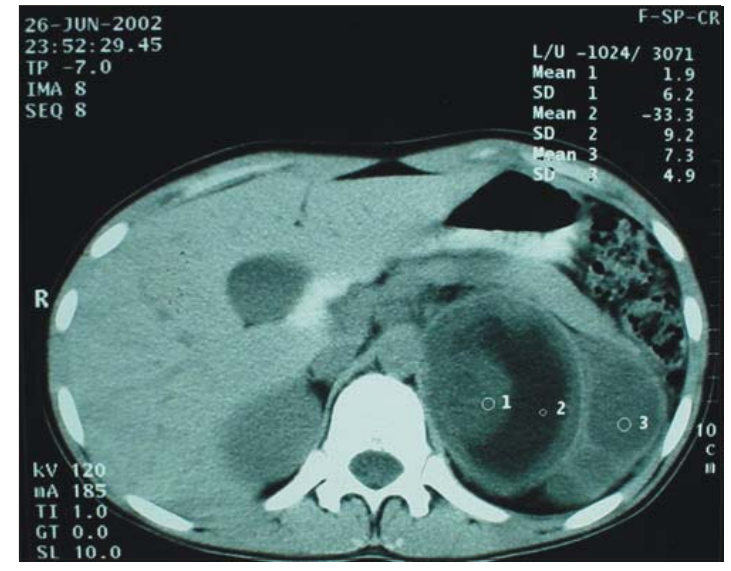

Figura 9. TAC: Teratoma maduro adrenal izquierdo, varón de 15 años.

El tamaño tumoral debe ser visto junto con otros factores para decidir si es tributario o no, de manejo laparoscópico. Los tumores grandes podrían ser tratados por vía laparoscópica, si se cuenta con el entrenamiento y logística adecuados (incluyendo HALS). El tamaño tumoral puede variar según se considere la tomografía, el acto quirúrgico o el patólogo.

La técnica hoy en día está casi completamente estandarizada: colocación del paciente, distribución de trócares, maniobras de disección, ligadura vascular y extracción de la pieza

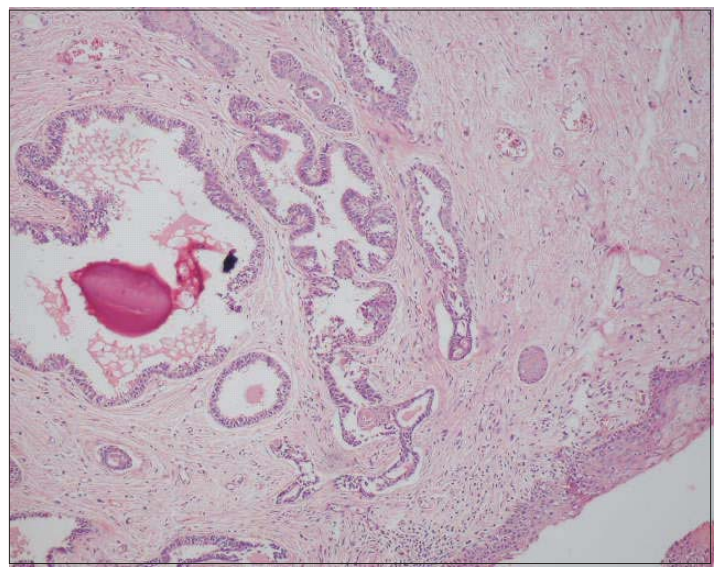

Figura 11. Microscopía: Teratoma maduro adrenal izquierdo, varón de 15 años.

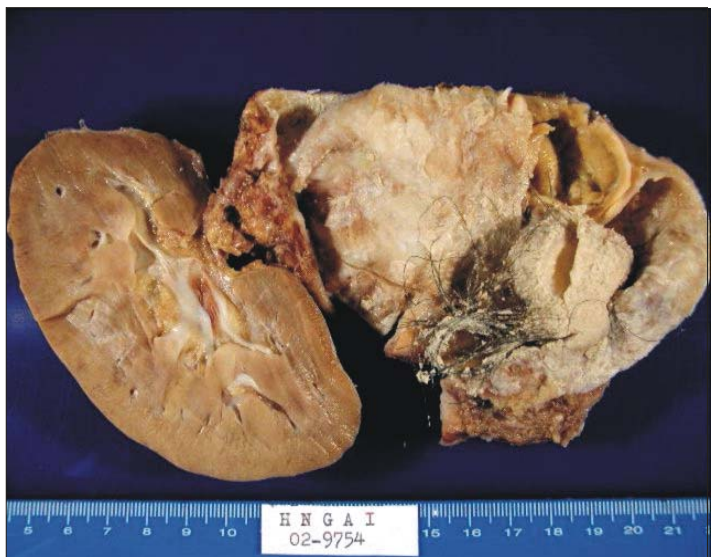

Figura 10. Macroscopía: Teratoma maduro adrenal izquierdo, varón de 15 años.

operatoria. Algunas patologías, como por ejemplo el feocromocitoma, exigen de conocer el manejo pre y trans operatorio para obtener un adecuado resultado.

\section{REFERENCIAS BIBLIOGRÁFICAS}

1. Gagner M, Lacroix A, Bolte E. Laparoscopic adrenalectomy in Cushing's syndrome and pheochromocytoma. N Engl J Med. 1992;327:1033.

2. Poulin E, Schiachta C, Burpee S, Pace K, Mamazza J. Laparoscopic adrenalectomy: pathologic features determine outcome. J Can Chir. 2003;46:340-4.

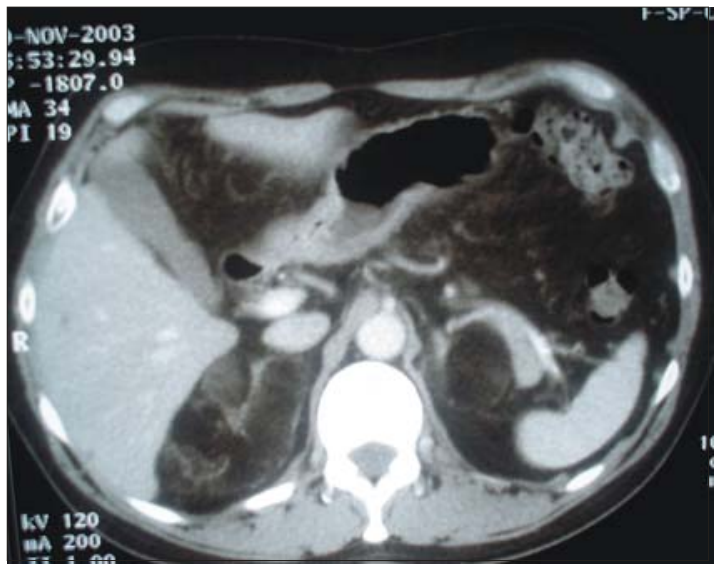

Figura 12. TAC: Mielolipoma bilateral, varón de 57 años. 
3. Gagner M, Pomp A, Heniford BT, Pharand D, Lacroix A. Laparoscopic adrenalectomy: lessons learned from 100 consecutive procedures. Ann Surg. 1997;226:238-46.

4. Imai T, Kikumori T, ohiwa M, Mase T, Funahashi H. A case-controlled study of laparoscopic compared with open lateral adrenalectomy. Am J Surg. 1999;178:50-4.

5. Brunt LM, Doherty GM, Norton JA, Soper NJ, Quasebarth MA, Moley JF. Laparoscopic adrenalectomy compared to open open adrenalectomy for benign adrenal neoplasms. J Am Coll Surg. 1996;183:1-10.

6. Lezoche E, Guerrieri M, Paganini AM, Feliciotti F, Zenobi $\mathrm{P}$, Antognini F. Laparoscopic adrenalectomy by the anterior transperitoneal approach: results of 108 operations in unselected cases. Surg Endosc. 2000;14:920-5.

7. Dackiw A, Lee J, Gagel R. Adrenal cortical carcinoma. World J Surg. 2001;25:914-26.

8. Barresi R, Prinz R. Laparoscopic adrenalectomy. Arch Surg. 1999;134:212-7.

9. Li M, Fitzgerald P. Iatrogenic pheochromocytomatosis: a previously unreported result of laparoscopic adrenalectomy. Surgery. 2001;130:1072-7.

10. Fernandez JM, Huescar AM. Primary leiomyosarcoma. A rare tumor of the adrenal gland. Arch Esp Urol. 1998;51(10):1029-31.

11. Thamboo TP, Liew LC, Raju GC. Adrenal leiomyosarcoma: a case report and literature review. Pathology. 2003;35(1):47-9.
12. Linos D, Kiriakopoulos AC, Tsakayannis DE, Theodoridou M, Chrousos G. Laparoscopic excision of bilateral primary adrenal leiomyosarcomas in a 14-year-old girl with acquired immunodeficiency syndrome (AIDS). Surgery. 2004;136(5):1098-100.

13. Matsui Y, Fujikawa K, Oka H, Fukuzawa S, Takeuchi H. Adrenal leiomyosarcoma extending into the right atrium. Intern J Urol. 2002;9:54-6.

14. Heniford B, Arca M, Walsh M. Laparoscopic adrenalectomy for cancer. Semin Surg Oncol. 1999;16:293-306.

15. Kebebew E, Siperstein A, Clark O. Results of laparoscopic adrenalectomy for suspected and unsuspected malignant adrenal neoplasms. Arch Surg. 2002;137:948-53.

16. Novitsky Y, Czerniach D, Kent W, Kercher, Perugini R, Kelly J, Litwin D. Feasibility of laparoscopic adrenalectomy for large adrenal masses. Surg Laparosc Endosc Percutan Tech. 2003;13:106-10.

Manuscrito recibido el 05 de diciembre de 2005 y aceptado para publicación el 20 diciembre de 2005.

Correspondencia: Dr. José De Vinatea De Cárdenas

San Ignacio de Loyola 476, Dpto 302

Lima 18, Perú

Correo-e:jomal@terra.com.pe 